\title{
Descripción de las características clínicas y la respuesta a tratamiento en pacientes con miastenia grave sin timoma sometidos a timectomía en una institución de alta complejidad de Cali, Colombia, 2010-2017
}

\author{
Description of the clinical characteristics and response to treatment in \\ patients with myasthenia gravis without thymoma undergoing thymectomy \\ in a tertiary level care institution in Cali, Colombia, 2010-2017
}

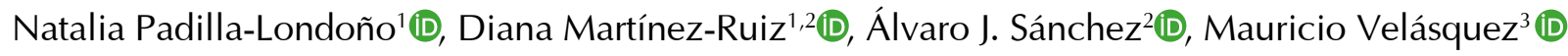

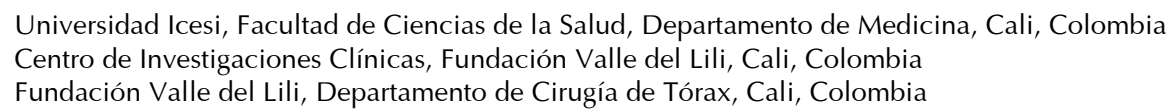

\section{Resumen}

Introducción. La miastenia grave es una enfermedad autoinmunitaria con una prevalencia mundial de 150 a 250 casos por I' ooo.ooo de habitantes. El tratamiento recomendado para la miastenia grave sin timoma es la timectomía total, la cual es la única alternativa de curación.

Métodos. Se llevó a cabo un estudio descriptivo y retrospectivo de una serie de casos de pacientes adultos con miastenia grave sin timoma sometidos a timectomía, durante el periodo de 2010 a 20I7. En el análisis estadístico descriptivo, se utilizaron frecuencias absolutas y porcentajes para las variables cualitativas y, para las variables cuantitativas, se utilizaron la mediana y el rango intercuartílico.

Resultados. Veintiocho pacientes con miastenia grave sin timoma se sometieron a timectomía desde el año 2010 hasta el 2017. Se categorizaron según la clasificación del estado posterior a la intervención de la Myasthenia Gravis Foundation of America y se evidenció que 4 (I4,3\%) pacientes presentaban remisión completa y el grado 3 de manifestaciones clínicas mínimas fue el más frecuente en I9 (67,9\%); 26 (92,9\%) tuvieron mejoría con respecto al cambio del estado clínico, en 2 (7,I \%) no se documentaron cambios y en ningún paciente hubo empeoramiento, exacerbación o muerte secundaria a la enfermedad.

Fecha de recibido: 11/12/2019 - Fecha de aceptación: 20/01/2020

Correspondencia: Diana Martínez, estadística, M. Sc., Centro de Investigaciones Clínicas, Fundación Valle del Lili, Carrera 98 № 18 49, Cali 760032, Colombia. Teléfono: (572) 331-9090, extensión 4022. Correo electrónico: diana.marcela.ru@fvl.org.co

Citar como: Padilla-Londoño N, Martínez-Ruiz D, Sánchez AJ, Velásquez M. Descripción de las características clínicas y la respuesta a tratamiento en pacientes con miastenia grave sin timoma sometidos a timectomía en una institución de alta complejidad de Cali, Colombia, 2010-2017. Rev Colomb Cir. 2020;35:391-7. https://doi.org/10.30944/20117582.121

Este es un artículo de acceso abierto bajo una Licencia Creative Commons - BY-NC-ND https://creativecommons.org/licenses/by-ncnd/4.0/deed.es 
Conclusiones. A lo largo de siete años se practicó timectomía a 28 pacientes con diagnóstico de miastenia grave sin timoma, aproximadamente, en el $15 \%$ de los pacientes hubo remisión completa, el grado 3 de manifestaciones mínimas fue el más frecuente y el $93 \%$ presentó mejoría de su estatus clínico.

Palabras clave: miastenia gravis; timo; timectomía; cirugía torácica; resultado del tratamiento.

\begin{abstract}
Introduction: Myasthenia gravis is an autoimmune disease with a worldwide prevalence of 150 to 250 cases per I,ooo,ooo inhabitants. The recommended treatment for myasthenia gravis without thymoma is total thymectomy, which is the only cure alternative.

Methods: A descriptive and retrospective study of a series of cases of adult patients with myasthenia gravis without thymoma undergoing thymectomy was conducted during the period from 20I0 to 20I7. In the descriptive statistical analysis, absolute frequencies and percentages were used for the qualitative variables, and for the quantitative variables the median and the interquartile range were used.

Results: Twenty-eight patients with myasthenia gravis without thymoma underwent thymectomy from 2010 to 2017. They were categorized according to the Myasthenia Gravis Foundation of America post-intervention status classification, and it was found that four (I4,3\%) patients had complete remission, and grade 3 of minimal clinical manifestations was the most frequent in 19 (67,9\%); 26 (92,9\%) had improvement with respect to the change of the clinical state, in two $(7, \mathrm{I} \%)$ no changes were documented and in no patient there was worsening, exacerbation or death secondary to the disease.

Conclusion: Over seven years, 28 patients diagnosed with myasthenia gravis without thymoma underwent thymectomy. Approximately in $15 \%$ of patients achieve complete remission, grade 3 of minimal manifestations was the most frequent and $93 \%$ presented improvement in their clinical status.
\end{abstract}

Key words: myasthenia gravis; thymus gland; thymectomy; thoracic surgery; treatment outcome.

\section{Introducción}

La miastenia grave es una enfermedad autoinmunitaria generada por la producción de anticuerpos contra los receptores activos de la acetilcolina localizados en la hendidura posináptica de la unión neuromuscular, que impide la despolarización y la generación de potenciales de acción, lo cual conlleva las principales manifestaciones clínicas de fatiga y debilidad muscular, que empeoran con la actividad diaria y mejoran con el reposo ${ }^{\mathrm{I}-3}$.

Mundialmente, la prevalencia anual es de I50 a 250 casos por I'ooo.0oo de habitantes; la enfermedad se presenta predominantemente en dos grupos etarios, según el sexo: uno entre los 20 y los 30 años en mujeres, y otro entre los 60 y los 70 años en hombres ${ }^{1,2}$. En el año 2002, en un estudio en Antioquia se estimó por primera vez la prevalencia de la miastenia grave en Colombia en 27,7 casos por I'ooo.0oo de habitantes. Esta cifra es baja con respecto a los datos mundiales, aunque concuerda con la información reportada en otros países tropicales donde, además, se describe una tendencia al incremento ${ }^{3,4}$.

Se ha demostrado que el timo contribuye a la producción de los anticuerpos que median la reacción autoinmunitaria presente en la miastenia grave. El $75 \%$ de los pacientes presenta anormalidades en esta glándula, en el Io \% específicamente, tumores tímicos o timomas, y en el $65 \%$, hiperplasia o centros germinales activos en el tejido tímico, a los cuales se les diagnostica miastenia grave sin timoma ${ }^{2,5,6}$. Por esto, entre los tratamientos planteados para la miastenia 
grave, se encuentra la timectomía total, la cual es especialmente beneficiosa para los pacientes con miastenia grave sin timoma; no obstante, la fisiopatología de esta asociación aún no ha sido completamente dilucidada 5 .

En múltiples estudios se plantea la timectomía como una intervención quirúrgica costo-efectiva, ya que, en un periodo de tres años, en los pacientes con miastenia grave sin timoma, se mejora la sintomatología, se disminuyen la necesidad de medicamentos inhibidores de la colinesterasa e inmunosupresores y se disminuyen los días de hospitalización para el manejo de las exacerbaciones de la enfermedad 7 .

El primer reporte que existe sobre la timectomía es de hace 75 años, aproximadamente, y, a partir de entonces, se han desarrollado múltiples estudios en los que se sugiere que, entre mayor cantidad de tejido tímico se retire, mejores son los resultados clínicos de la intervención ${ }^{8,9}$. Se han descrito diferentes técnicas quirúrgicas para la timectomía; se puede practicar mediante abordaje abierto, como la esternotomía, o con intervenciones menos invasivas, como la toracoscopia bilateral o la unilateral ${ }^{7,10}$. No existe evidencia clínica clara que indique el mejor tipo de abordaje quirúrgico en estos pacientes.

Por esta razón, el propósito de este estudio fue describir las características clínicas y la respuesta terapéutica en un seguimiento de, por lo menos, un año, desde el año 2010 al 20I7, en pacientes adultos con miastenia grave sin timoma sometidos a timectomía mediante estos tres diferentes abordajes quirúrgicos en la Fundación Valle del Lili, un hospital de referencia y de alta complejidad en el suroccidente de Colombia.

\section{Métodos}

Contexto. El estudio se llevó a cabo en una institución en la región suroccidental de Colombia, enfocada en la atención de pacientes de alta complejidad. En esta institución, la timectomía es practicada desde el año $20 ı$ por un cirujano de tórax, con gran experiencia en el manejo de esta enfermedad.

Tipo de estudio. Se hizo un estudio descriptivo y retrospectivo de todos los pacientes adultos con diagnóstico de miastenia grave sin timoma confirmada mediante histología, que fueron sometidos a timectomía durante los años 2010 a 20I7. Se excluyeron los pacientes intervenidos con técnicas quirúrgicas diferentes a las tres descritas en el presente estudio.

Recolección y procesamiento de datos. De las historias clínicas físicas y electrónicas, se recolectó la información de las variables demográficas y clínicas, el tipo y las características de la cirugía, y de la respuesta terapéutica, con un seguimiento de, por lo menos, un año y, en algunos pacientes hasta por siete años, después de la intervención quirúrgica.

Medición de resultados. Los pacientes se categorizaron según la clasificación del estado posquirúrgico de la Myasthenia Gravis Foundation of America, en la cual se describe la remisión completa, cuando no se han presentado signos ni síntomas de miastenia grave en el último año, sin ningún tratamiento para esta enfermedad durante el mismo periodo; además, la remisión farmacológica, cuando no se han presentado signos ni síntomas de miastenia graves en el último año, pero se ha requerido algún medicamento para la enfermedad, diferente a los inhibidores de la colinesterasa.

También, se describen las manifestaciones mínimas, cuando los pacientes no presentan síntomas de limitaciones funcionales secundarios a la miastenia grave, aunque sí alguna debilidad al examen físico. Estas se dividen en: MM-o, cuando los pacientes no han recibido tratamiento para la miastenia grave en el último año; MM-I, cuando continúan recibiendo inmunosupresores, pero no han requerido inhibidores de la colinesterasa ni otro tratamiento sintomático; $\mathrm{MM}-2$, cuando han recibido bajas dosis de inhibidores de colinesterasa (<ا2O $\mathrm{mg}$ de piridostigmina al día) en el último año; y MM-3, cuando han recibido inhibidores de la colinesterasa u otros tratamientos sintomáticos e inmunosupresores, en el último año.

Por último, en esta clasificación se incluye el cambio del estado clínico que puede calificarse como: mejoría, cuando se presenta una disminución significativa de las manifestaciones clínicas 
previas a la cirugía o una reducción sustancial en la terapia farmacológica previa a la cirugía; sin cambios, cuando no hay diferencias significativas con respecto a las manifestaciones clínicas y la terapia farmacológica previas a la cirugía; empeoramiento, cuando hay un aumento sustancial de las manifestaciones clínicas y de la necesidad de fármacos, en comparación con el tiempo previo a la cirugía; exacerbación, cuando se han cumplido los criterios de remisión completa, de remisión farmacológica o de manifestaciones mínimas, pero se presenta empeoramiento; y por último, la muerte secundaria a la miastenia grave por complicaciones en la terapia farmacológica o durante los 30 días después de la timectomía.

Análisis estadístico. Los datos de interés se registraron en el software BDClinic. Las variables cuantitativas se describieron por medio de la mediana y el rango intercuartílico (RIQ), dado que no tenían distribución normal verificada con la prueba de Shapiro-Wilk, y las variables cualitativas se presentaron como frecuencias absolutas y relativas.

\section{Resultados}

Durante los años 2010 a 2017 , a 28 pacientes adultos con miastenia grave sin timoma se les practicó la timectomía con una de tres técnicas quirúrgicas diferentes en la Fundación Valle del Lili, un hospital de alta complejidad. En la tabla I se presentan las características demográficas y clínicas de los pacientes; la mitad de ellos tenía 40 años de edad al momento de la cirugía y, en su mayoría, eran hombres.

Los síntomas más frecuentes fueron ptosis palpebral y debilidad en los miembros superiores, seguidos de debilidad en los miembros inferiores; la duración de los síntomas hasta el diagnóstico fue mayor de tres meses. Respecto al manejo clínico previo a la timectomía, todos los pacientes habían recibido inhibidores de la colinesterasa y, aproximadamente, el $80 \%$ recibió prednisona como medicamento inmunosupresor.

El abordaje quirúrgico más frecuente fue mediante toracoscopia en $20(7 \mathrm{I}, 4 \%)$ pacientes, de las cuales I 4 (50,0 \%) fueron derechas y $6(2 \mathrm{I}, 4 \%)$ fueron bilaterales; en $8(28,6 \%)$, se utilizó la esternotomía. La esternotomía era la técnica quirúrgica preferida hasta el año 20I3; posteriormente, entre el 2013 y el 20I4, se utilizó la toracoscopia bilateral y, finalmente, la toracoscopia derecha a partir del 2014 y hasta el 20I7. La mediana de edad de los pacientes con esternotomía fue de 45,5 años (rango: 42 a 58,5), en los de toracoscopia bilateral, fue 33,5 años (rango: I9 a 7I) y, en los de toracoscopia derecha, de 32 años (rango: 24 a 53).

En la tabla 2 se presentan las complicaciones y resultados de los pacientes según el tipo de cirugía. La toracoscopia bilateral fue el procedimiento de mayor duración y, la toracoscopia derecha, el de menor duración. El tiempo promedio de hospitalización fue de cinco días. En I9 de

Tabla 1. Características demográficas y clínicas de los pacientes

\begin{tabular}{ll}
\hline \multicolumn{1}{c}{ Características } & \multicolumn{1}{c}{$\mathbf{n ~ ( \% )}$} \\
\hline Edad al momento de la cirugía* & $40(23,5-58,5)$ \\
Femenino & $12(42,9)$ \\
Masculino & $16(57,1)$ \\
Síntomas & \\
Duración de síntomas hasta el diagnóstico \\
$\leq 3$ meses & $2(7,1)$ \\
>3 meses & $26(92,9)$ \\
Duración de síntomas hasta la cirugía & \\
$\leq 3$ meses & $1(3,6)$ \\
>3 meses & $27(96,4)$ \\
Ptosis palpebral & $22(78,6)$ \\
Disfagia & $14(50,0)$ \\
Diplopía & $11(39,3)$ \\
Disartria & $9(32,1)$ \\
Disnea & $8(28,6)$ \\
Debilidad de las extremidades & \\
Superior & $16(57,1)$ \\
Inferior & $14(50,0)$ \\
Tratamiento & \\
Inhibidores de la colinesterasa & $28(100)$ \\
Prednisona & $22(78,6)$ \\
Plasmaféresis & $10(35,7)$ \\
Ig IV & $9(32,1)$ \\
Otros inmunosupresores & $16(57,1)$ \\
Azatioprina & $11(68,8)$ \\
Deflazacort & $3(18,8)$ \\
Micofenolato de mofetilo & $5(31,3)$ \\
\hline
\end{tabular}

* Mediana (rango intercuartílico) 
Tabla 2. Complicaciones y resultados según el tipo de cirugía

\begin{tabular}{|c|c|c|c|c|c|c|c|c|}
\hline \multirow[t]{2}{*}{ Variables } & \multicolumn{2}{|c|}{$\begin{array}{l}\text { Esternotomía } \\
\quad(n=8)\end{array}$} & \multicolumn{2}{|c|}{$\begin{array}{l}\text { Toracoscopia } \\
\text { bilateral }(n=6)\end{array}$} & \multicolumn{2}{|c|}{$\begin{array}{c}\text { Toracoscopia } \\
\text { derecha }(n=14)\end{array}$} & \multicolumn{2}{|c|}{ Total $(n=28)$} \\
\hline & $\mathbf{n}$ & $\%$ & $\mathbf{n}$ & $\%$ & $\mathbf{n}$ & $\%$ & $\mathbf{n}$ & $\%$ \\
\hline Tiempo de cirugía (minutos) ${ }^{*}$ & \multicolumn{2}{|c|}{$160(150-170)$} & \multicolumn{2}{|c|}{$235(210-255)$} & \multicolumn{2}{|c|}{$100(85-130)$} & \multicolumn{2}{|c|}{$150(90-180)$} \\
\hline \multicolumn{9}{|l|}{ Complicaciones posoperatorias } \\
\hline Ninguna & 5 & 62,5 & 5 & 83,3 & 9 & 64,3 & 19 & 67,9 \\
\hline Crisis miasténica & 1 & 12,5 & 1 & 16,7 & 2 & 14,3 & 4 & 14,3 \\
\hline Neumonía & 1 & 12,5 & 0 & 0,0 & 1 & 7,1 & 2 & 7,1 \\
\hline Lesión nerviosa permanente & 0 & 0 & 0 & 0,0 & 1 & 7,1 & 1 & 3,6 \\
\hline Neumotórax & 0 & 0 & 0 & 0,0 & 1 & 7,1 & 1 & 3,6 \\
\hline Derrame pleural & 1 & 12,5 & 0 & 0,0 & 0 & 0,0 & 1 & 3,6 \\
\hline Tiempo de hospitalización* & \multicolumn{2}{|c|}{$5,5(4,5-10)$} & \multicolumn{2}{|c|}{$4(4-4)$} & \multicolumn{2}{|c|}{$5(4-12)$} & \multicolumn{2}{|c|}{$5(4-9)$} \\
\hline Tiempo en $\mathrm{UCl}^{*}$ & \multicolumn{2}{|c|}{$4,5(2,5-7,5)$} & \multicolumn{2}{|c|}{$2,5(2-4)$} & \multicolumn{2}{|c|}{$3(2-8)$} & \multicolumn{2}{|c|}{$3,5(2-6)$} \\
\hline Número de controles posoperatorios* & \multicolumn{2}{|c|}{$3(2-4)$} & \multicolumn{2}{|c|}{$2(1-5)$} & \multicolumn{2}{|c|}{$2(1-3)$} & \multicolumn{2}{|c|}{$2(1-4)$} \\
\hline \multicolumn{9}{|l|}{ Resultados } \\
\hline \multicolumn{9}{|l|}{ Estado } \\
\hline Vivo & 5 & 62,5 & 6 & 100,0 & 11 & 78,6 & 22 & 78,6 \\
\hline Sin seguimiento & 3 & 37,5 & 0 & 0,0 & 3 & 21,4 & 6 & 21,4 \\
\hline \multicolumn{9}{|l|}{ Remisión } \\
\hline Completa & 2 & 25 & 1 & 16,7 & 1 & 7,1 & 4 & 14,3 \\
\hline Farmacológica & 0 & 0 & 0 & 0 & 0 & 0 & 0 & 0 \\
\hline \multicolumn{9}{|l|}{ Manifestaciones mínimas } \\
\hline Grado 0 & 2 & 25 & 1 & 16,7 & 1 & 7,1 & 4 & 14,3 \\
\hline Grado 1 & 0 & 0 & 1 & 16,7 & 0 & 0,0 & 1 & 3,6 \\
\hline Grado 2 & 0 & 0 & 1 & 16,7 & 3 & 21,4 & 4 & 14,3 \\
\hline Grado 3 & 6 & 75 & 3 & 50,0 & 10 & 71,4 & 19 & 67,9 \\
\hline \multicolumn{9}{|l|}{ Cambio de estatus } \\
\hline Mejoría & 7 & 87,5 & 6 & 100,0 & 14 & 100,0 & 26 & 92,9 \\
\hline Sin cambios & 1 & 12,5 & 0 & 0,0 & 1 & 7,1 & 2 & 7,1 \\
\hline
\end{tabular}

* Mediana (rango intercuartílico)

los 28 pacientes no presentaron complicaciones posoperatorias y la más frecuente fue la crisis miasténica, que se presentó en cuatro casos.

Respecto a los resultados clínicos en el seguimiento posterior a la intervención quirúrgica, 4 (I4,3\%) pacientes presentaron remisión completa, a 2 de los cuales se les practicó esternotomía y, a los otros 2, toracoscopia. En los tres tipos de abordajes quirúrgicos, el grado más frecuente de manifestaciones mínimas fue el 3. Con relación al cambio en el estado clínico después de la intervención, 26 (92,9\%) de los pacientes presentaron mejoría y, en los 20 so- metidos a toracoscopia, derecha o bilateral, esta cifra alcanzó el Ioo \%.

\section{Discusión}

Este estudio retrospectivo de 28 pacientes con diagnóstico confirmado de miastenia grave sin timoma, muestra que la timectomía en nuestra institución es un procedimiento seguro ya que ningún paciente falleció a causa de la intervención quirúrgica, se requirieron pocos días de hospitalización y la frecuencia de complicaciones posquirúrgicas fue baja. Este hallazgo concuerda con 
múltiples estudios, en los cuales la mortalidad posquirúrgica de la timectomía fue de o a I \%, con una estadía hospitalaria menor de cinco días y con evidencia de que las complicaciones posquirúrgicas cada vez más tienden a disminuir ${ }^{\mathrm{II-1} 3}$.

Se encontró que, independientemente del abordaje quirúrgico, la mayoría de los pacientes presentaron mejoría en su estado clínico en el control posquirúrgico, por lo menos, un año después del procedimiento. Según la clasificación del estado posterior a la intervención de la Myasthenia Gravis Foundation of America, esto significa que hubo un descenso significativo de las manifestaciones clínicas o una reducción sustancial de la terapia farmacológica previas a la intervención quirúrgica. Nuestros resultados fueron superiores a los de múltiples estudios, en los cuales del $7 \mathrm{I}$ al $87 \%$ de los pacientes con miastenia grave sin timoma presentó mejoría de su estado clínico después de la timectomía ${ }^{\text {14-16 }}$.

Con relación a la remisión completa, o sea, cuando los pacientes no han presentado signos ni síntomas de miastenia grave y no han recibido ninguna terapia en el último año, en el presente estudio el porcentaje obtenido fue más bajo que los descritos a nivel mundial, los cuales oscilan entre el $19 \%$ y el $75 \%$ de pacientes, con seguimiento de Io y 15 años, respectivamente ${ }^{8,15}$. Esto podría deberse a la diferencia en el tiempo de seguimiento.

Se ha evidenciado que el manejo quirúrgico con timectomía beneficia a los pacientes con miastenia grave sin timoma y, aunque en el presente estudio no se realizaron comparaciones con el tratamiento farmacológico exclusivo, diferentes autores han demostrado que la timectomía es superior al mismo. En el más reciente estudio clínico aleatorizado en pacientes con miastenia grave sin timoma, se evidenció que la timectomía mejoraba los resultados clínicos en un periodo de tres años, con disminución de la necesidad de medicamentos inmunosupresores, de la sintomatología y de las hospitalizaciones por exacerbaciones de la enfermedad ${ }^{17}$.

Una limitación de este estudio fue el subregistro de la información de las variables de in- terés, debido a que se recolectó de un sistema físico y uno electrónico de historias clínicas, en el cual podían faltar algunos datos. Se recomienda realizar futuros estudios en nuestro medio con un diseño epidemiológico y la rigurosidad estadística necesaria que permitan ampliar el conocimiento sobre las características y el impacto de la miastenia grave, como las opciones terapéuticas, la evolución postquirúrgica y los resultados clínicos.

\section{Conclusiones}

A lo largo de los siete años del periodo de estudio en nuestra institución, se sometió a timectomía a 28 pacientes con diagnóstico de miastenia grave sin timoma y el abordaje quirúrgico más frecuente fue la toracoscopia derecha. Ninguno de los pacientes falleció y hubo complicaciones posoperatorias en menos del $35 \%$. Después de un año del procedimiento quirúrgico, las manifestaciones mínimas de grado 3 fueron las más frecuentes, aproximadamente el $15 \%$ de los pacientes lograron la remisión completa y $92,9 \%$ presentaron mejoría en su estatus clínico.

\section{Agradecimientos}

Agradecemos al Centro de Investigaciones Clínicas de la Fundación Valle del Lili.

\section{Cumplimiento de normas éticas}

Consideraciones éticas. El protocolo para este estudio fue aprobado por el Comité de Ética e Investigación Biomédica de la institución (protocolo No. 1247).

Consentimiento informado. Este estudio es una revisión de historias clínicas y, como tal, no hubo necesidad de un consentimiento informado.

Conflicto de intereses. Los autores declaran no tener conflicto de intereses.

Financiación. Este estudio fue financiado con recursos de la Fundación Valle del Lili, Cali, Colombia. 


\section{Referencias}

I. Gilhus NE. Myasthenia gravis. N Engl J Med. 20I6;375:2570-8I. https://doi.org/Io.IO56/NEJMraI602678

2. Drachman DB. Miastenia grave y otras enfermedades de la unión neuromuscular. En: Serrano H, Merediz J, Sánchez C, editores. Harrison. Principios de Medicina Interna. I8 edición. Ciudad de México: McGraw-Hill Education; 2012. p. 3480-6.

3. Tapias-Vargas L, Tapias-Vargas LF, Tapias L. Miastenia gravis y el timo: pasado, presente y futuro. Rev Colomb Cir. 20II;24:269-82.

4. Sánchez J, Uribe C, Franco A, Jiménez M, Arcos-Burgos M, Palacio L. Prevalencia de la miastenia grave en Antioquia, Colombia. Rev Neurol. 2002;34:IOIO-2.

5. Nieto I, Robledo J, Pajuelo M, Montes JAR, Girón JG, Alonso JG. Prognostic factors for myasthenia gravis treated by thymectomy: Review of 6I cases. Ann Thorac Surg. 1999;67:1568-7I.

6. Werneck L, Cunha F, Scola R. Myasthenia gravis: A retrospective study comparing thymectomy to conservative treatment. Acta Neurol Scand. 2000;IOI:4I-6.

7. Jaretzki A, Steinglass KM, Sonett JR. Thymectomy in the management of myasthenia gravis. Semin Neurol. 2004;24:49-59.

8. Kaufman AJ, Palatt J, Sivak M, Raimondi P, Lee DS, Wolf A. Thymectomy for myasthenia gravis: Complete stable remission and associated prognostic factors in over IOoo cases. Semin Thorac Cardiovasc Surg. 2016;28:56I-8. https://doi.org/ro.I053/j.semtcvs.2016.04.002

9. Jaretzki AI. Thymectomy for myasthenia gravis: Analysis of controversies - Patient management. Neurologist. 2003;9:77-92.
Io. Jaretzki A, Barohn R, Ernstoff R, Kaminski HJ, Keesey JC, Penn AS. Myasthenia gravis: Recommendations for clinical research standards. Neurology. 2000;55:16-23.

II. Luo Y, Pan D, Chen F, Zhu M, Wang J, Zhang M. Effectiveness of thymectomy in non-thymomatous myasthenia gravis: A systematic review. J Huazhong Univ Sci Technol Med Sci. 2014;34:942-9. https://doi.org/IO.I007/sII596-OI4-I378-X

I2. Bachmann K, Burkhardt D, Schreiter I, Kaifi J, Schurr $\mathrm{P}$, Busch C. Thymectomy is more effective than conservative treatment for myasthenia gravis regarding outcome and clinical improvement. Surgery. 2009;145:392-8. https://doi.org/I0.IoI6/j.surg.2008.II.009

I3. Papatestas AE, Genkins G, Kornfeld P, Eisenkraft JB, Fagerstrom RP, Pozner J. Effects of thymectomy in myasthenia gravis. Ann Surg. 1987;206:79-88.

I4. Perrot M De, Licker M, Spiliopoulos A. Factors influencing improvement and remission rates after thymectomy for myasthenia gravis. Respiration. 200I;68:60I-5.

I5. El-Medany Y, Hajjar W, Essa M, Al-Kattan K, Hariri Z, Ashour M. Predictors of outcome for myasthenia gravis after thymectomy. Asian Cardiovasc Thorac Ann. 2003;II:323-7.

I6. Venuta F, Rendina EA, Giacomo TD, Rocca G, Antonini G, Ciccone A. Thymectomy for myasthenia gravis: A 27-year experience I. Eur J Cardio-thoracic Surg. 1999;15:62I-5.

17. Barboi A, Amato A, Shaibani A, Katirji B, Lecky BRF, Buckley C. Randomized trial of thymectomy in myasthenia gravis. N Engl J Med. 20I6;375:5II-22. https://doi.org/Io.I056/NEJMoar602489 